\title{
Derivation of a non-local interfacial Hamiltonian for short-ranged wetting: I. Double-parabola approximation
}

\author{
A O Parry ${ }^{1}$, C Rascón ${ }^{2}$, N R Bernardino ${ }^{1}$ and J M Romero-Enrique ${ }^{3}$ \\ ${ }^{1}$ Department of Mathematics, Imperial College London, London SW7 2BZ, UK \\ 2 Grupo Interdisciplinar de Sistemas Complejos (GISC), Departamento de Matemáticas, \\ Universidad Carlos III de Madrid, 28911 Leganés, Madrid, Spain \\ ${ }^{3}$ Departamento de Física Atómica, Molecular y Nuclear, Universidad de Sevilla, Apartado de \\ Correos 1065, 41080 Seville, Spain
}

Received 9 May 2006

Published 28 June 2006

Online at stacks.iop.org/JPhysCM/18/6433

\begin{abstract}
We derive a non-local effective interfacial Hamiltonian model for short-ranged wetting phenomena using a Green's function method. The Hamiltonian is characterized by a binding potential functional and is accurate to exponentially small order in the radii of curvature of the interface and the bounding wall. The functional has an elegant diagrammatic representation in terms of planar graphs which represent different classes of tube-like fluctuations connecting the interface and wall. For the particular cases of planar, spherical and cylindrical interfacial (and wall) configurations, the binding potential functional can be evaluated exactly. More generally, the non-local functional naturally explains the origin of the effective position-dependent stiffness coefficient in the smallgradient limit.
\end{abstract}

\section{Introduction}

In a recent letter [1], it was argued that many of the long-standing problems in the fluctuation theory of three-dimensional short-ranged wetting [2], including the order of the critical wetting transition and the size of the asymptotic critical regime [3-8], may be overcome using a nonlocal effective interfacial Hamiltonian. This model may also be used to study adsorption at non-planar walls, and similarly resolves known difficulties associated with local effective Hamiltonian treatments $[9,10]$.

In the present series of articles, we give full details of how such a non-local interfacial Hamiltonian for wetting may be explicitly derived from an underlying Landau-GinzburgWilson (LGW) model using a Green's function method. The techniques employed are closely related to multiple-reflection expansion methods used for other problems [11, 12], including Kac's famous question concerning the eigenvalue spectra of differently shaped drums [13]. 
The derivation given here is essentially an improved version of the recipe introduced by Fisher and Jin (FJ) [6] with the advantage that it identifies the interfacial Hamiltonian to exponentially (as opposed to algebraically) accurate order in the relevant radii of curvature, and reveals the non-local nature of the interfacial Hamiltonian. Our central result is that the Hamiltonian is characterized by a binding potential functional which can be written as an elegant diagrammatic expansion

$$
w=a_{1} \longrightarrow+b_{1} \longrightarrow
$$

in terms of planar graphs, which represent different classes of tube-like fluctuations connecting the unbinding interface and the wall (see below).

Our presentation is split into two papers: in this first article, we consider the derivation of the interfacial model from an LGW theory which has fixed order parameter (magnetization) at the bounding wall, rather than coupling to a surface field and enhancement, and also within the so-called double-parabola (DP) approximation to the potential modelling of the bulk coexistence $[6,14]$. Both of these choices make the derivation of the model most transparent without influencing the underlying physics. The DP approximation also has the advantage that one can identify all the contributions to the binding potential functional and classify all the higher-order diagrams. In paper II, we extend the Green's function method to allow for perturbations about the DP approximation, the inclusion of squared Laplacian terms in the Hamiltonian, and also the case of coupling to a surface field and enhancement.

Our paper is arranged as follows. In the next section, we present some necessary background material, in particular the FJ perturbative derivation of a local interfacial Hamiltonian with a position-dependent binding potential function and stiffness coefficient. Following this, we present an alternative (non-perturbative) derivation using Green's functions, identifying the non-local form of the Hamiltonian and binding potential functional. The diagrammatic interpretation of this functional is discussed in some detail. We show how, for planar, spherical and cylindrical interfacial (and wall) configurations, the diagrams can be evaluated and resummed to recover the known form of the binding potential function in these geometries. Finally, we demonstrate that, in the small-gradient limit, the non-local model reduces to the FJ Hamiltonian, and, in particular, how an effective position-dependent stiffness emerges from the underlying non-locality. A connection with exact statistical mechanical sumrules is also made.

\section{Background theory: the Fisher-Jin derivation}

The derivation of the non-local model begins by following the scheme set out by FJ, who were first to systematically consider the process of integrating out degrees of freedom from a microscopic model. For the latter, FJ use the continuum LGW Hamiltonian

$$
H_{\mathrm{LGW}}[m]=\int \mathrm{d} \mathbf{r}\left\{\frac{1}{2}(\nabla m)^{2}+\Delta \phi(m)\right\}
$$

based on a magnetization-like order parameter $m(\mathbf{r})$. A potential $\phi(m)$ models the bulk coexistence of phases $\alpha$ and $\beta$ with order parameters $-m_{0}$ and $+m_{0}$, respectively (which, for simplicity, we assume exhibit Ising symmetry). The shifted potential $\Delta \phi(m)=\phi(m)-\phi\left(m_{0}\right)$ conveniently substracts the bulk contribution to the free energy (proportional to the volume). For wetting phenomena, it is believed that a DP approximation suffices to capture the critical singularities, and to this end we write (in zero bulk field)

$$
\Delta \phi(m)=\frac{\kappa^{2}}{2}\left(|m|-m_{0}\right)^{2}
$$




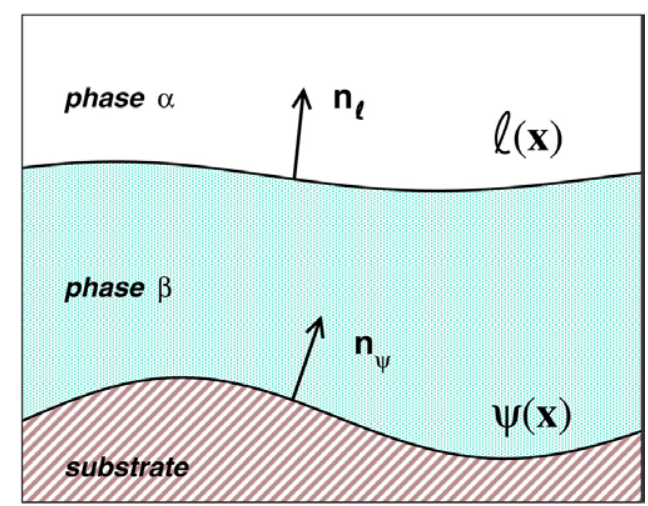

Figure 1. Schematic diagram of an interfacial configuration $\ell(\mathbf{x})$ of an adsorbed layer of phase $\beta$ on a substrate $\psi(\mathbf{x})$. Their respective normal vectors $\mathbf{n}_{\ell}$ and $\mathbf{n}_{\psi}$ are also shown.

(This figure is in colour only in the electronic version)

where $\kappa$ is the inverse bulk correlation length. We shall only consider the form of the interfacial model at bulk coexistence although, within the DP approximation, it is a straightforward exercise to extend the calculation to non-zero field.

We suppose that the system is bounded by a wall described by a height function $\psi=\psi(\mathbf{x})$ which is often conveniently measured above some plane with parallel displacement $\mathbf{x}=(x, y)$ (see figure 1). The most commonly studied example is the planar wall, for which $\psi=0$, although other pertinent examples are spheres, cylinders, and wedges. We suppose that the magnetization on the boundary is fixed:

$$
m(\mathbf{r})=m_{1}, \quad \text { for } \mathbf{r}=(\mathbf{x}, \psi(\mathbf{x})) .
$$

Without loss of generality, we assume that $m_{1}>0$, so the wetting layer forms at the wall- $\alpha$ interface, for which the bulk magnetization is $-m_{0}$. This choice of fixed boundary condition is easiest to implement using the method discussed here and allows the non-local nature of the interfacial model to be derived most cleanly. We emphasize that this does not influence the physics of the critical and complete wetting transitions. Varying $m_{1}$ at fixed temperature $T$ induces a (critical) wetting transition in exactly the same way that varying the surface field does in the LGW model with a surface potential. The mean-field (MF) critical wetting phase boundary, as defined for the planar wall- $\alpha$ interface, is readily shown to be $m_{1}=m_{0}$ (see below). At the MF level (and beyond, in three dimensions), $m_{0}-m_{1}$ is the relevant scaling field controlling the continuous divergence of the equilibrium wetting film thickness, together with parallel and perpendicular correlation lengths and the associated vanishing of the contact angle. How this MF critical wetting scenario is changed by interfacial fluctuation effects has been the topic of much debate in the literature [2-8] and is an essential application of the nonlocal model.

FJ introduced a number of definitions of the collective co-ordinate defining the interfacial configuration. The most convenient one to use, and the one adopted in the present paper, is a crossing criterion in which one identifies the interface as the surface of iso-magnetization at which the order parameter is constrained to be zero. Thus we consider constrained magnetization profiles for which

$$
m(\mathbf{r})=0 \quad \text { for } \mathbf{r}=(\mathbf{x}, \ell(\mathbf{x})),
$$

where $\ell(\mathbf{x})$ is the interfacial height (see figure 1). 
The interfacial Hamiltonian is formally defined via a partial trace over Boltzmann weighted configurations which respect the crossing criterion. A saddle point evaluation of the constrained sum leads to the FJ identification

$$
H[\ell, \psi]=H_{\mathrm{LGW}}\left[m_{\Xi}(\mathbf{r})\right]-F_{w \beta}[\psi]
$$

where we have subtracted a surface term corresponding to the excess free energy of the wall- $\beta$ interface $F_{w \beta}[\psi]$, which is explicitly determined in our calculation. In the above identification, $m_{\Xi}$ is the constrained profile that minimizes the LGW model subject to the crossing criterion and boundary condition. Within the DP approximation, this satisfies the Helmholtz equations

$$
\nabla^{2} m_{\Xi}= \begin{cases}\kappa^{2}\left(m_{\Xi}-m_{0}\right), & m_{\Xi}>0 \\ \kappa^{2}\left(m_{\Xi}+m_{0}\right), & m_{\Xi}<0\end{cases}
$$

with appropriate boundary conditions in the bulk and at the interface and the wall.

Before we present our derivation of a non-local interfacial model, it is worthwhile recalling some features of the original FJ derivation for planar substrates $(\psi=0)$. The FJ derivation is a perturbative one based on the properties of the planar constrained profile $m_{\pi}(z ; \ell)$. This satisfies the second-order ODEs

$$
\frac{\partial^{2} m_{\pi}}{\partial z^{2}}= \begin{cases}\kappa^{2}\left(m_{\pi}-m_{0}\right), & m_{\pi}>0 \\ \kappa^{2}\left(m_{\pi}+m_{0}\right), & m_{\pi}<0\end{cases}
$$

together with the boundary conditions $m_{\pi}(0 ; \ell)=m_{1}, m_{\pi}(\ell ; \ell)=0$ and $m_{\pi}(\infty ; \ell)=-m_{0}$. The planar constrained profile determines the binding potential $W_{\pi}(\ell)$, defined as the excess free energy per unit area of a constrained wetting layer with uniform (constrained) film thickness $\ell$. Within the DP approximation, $m_{\pi}(z ; \ell)$ is trivially determined. For $z \geqslant \ell$, this is particularly simple,

$$
m_{\pi}(z ; \ell)=-m_{0}\left(1-\mathrm{e}^{-\kappa(z-\ell)}\right)
$$

whilst within the region of interest $(0 \leqslant z \leqslant \ell)$

$$
\delta m_{\pi}(z ; \ell)=\frac{\delta m_{1}+m_{0} \mathrm{e}^{-\kappa \ell}}{1-\mathrm{e}^{-2 \kappa \ell}} \mathrm{e}^{-\kappa z}-\frac{m_{0}+\delta m_{1} \mathrm{e}^{-\kappa \ell}}{1-\mathrm{e}^{-2 \kappa \ell}} \mathrm{e}^{-\kappa(\ell-z)}
$$

where we have written $\delta m_{\pi}(z, \ell)=m_{\pi}(z, \ell)-m_{0}$, and $\delta m_{1}=m_{1}-m_{0}$. The two exponential terms $\mathrm{e}^{-\kappa(\ell-z)}$ and $\mathrm{e}^{-\kappa z}$ represent the tails of the (planar) $\alpha-\beta$ and wall- $\beta$ interfacial profiles with coefficients chosen to match the boundary conditions. For later purposes, it is useful to expand these coefficients and write

$$
\begin{aligned}
\delta m_{\pi}(z ; \ell)=\mathrm{e}^{-\kappa z}\left(\delta m_{1}+m_{0} \mathrm{e}^{-\kappa \ell}+\delta m_{1} \mathrm{e}^{-2 \kappa \ell}+\cdots\right) & \\
& -\mathrm{e}^{-\kappa(\ell-z)}\left(m_{0}+\delta m_{1} \mathrm{e}^{-\kappa \ell}+m_{0} \mathrm{e}^{-2 \kappa \ell}+\cdots\right) .
\end{aligned}
$$

By setting $z=0$ or $z=l$, one can see the term-by-term cancellations in the two series required to satisfy the crossing criterion and fixed surface magnetisation. The physical significance of the terms in this expansion will become apparent later. As noted above, the planar constrained profile determines the binding potential

$$
W_{\pi}(\ell)=\int \mathrm{d} z\left\{\frac{1}{2}\left(\frac{\partial m_{\pi}}{\partial z}\right)^{2}+\Delta \phi(m)\right\}-\Sigma_{w \beta}-\Sigma_{\alpha \beta}
$$

where $\Sigma_{\alpha \beta}$ and $\Sigma_{w \beta}$ are the tensions of the separate $\alpha \beta$ and wall- $\beta$ interfaces. Within the DP approximation, $\Sigma_{\alpha \beta}=\kappa m_{0}^{2}, \Sigma_{w \beta}=\kappa \delta m_{1}^{2} / 2$, and the binding potential is given by

$$
W_{\pi}(\ell)=2 \kappa \delta m_{1} m_{0} \frac{\mathrm{e}^{-\kappa \ell}}{1-\mathrm{e}^{-2 \kappa \ell}}+\left(\kappa m_{0}^{2}+\kappa \delta m_{1}^{2}\right) \frac{\mathrm{e}^{-2 \kappa \ell}}{1-\mathrm{e}^{-2 \kappa \ell}}
$$


which is usually expanded, keeping only the two leading order terms:

$$
W_{\pi}(\ell)=2 \kappa \delta m_{1} m_{0} \mathrm{e}^{-\kappa \ell}+\left(\kappa m_{0}^{2}+\kappa \delta m_{1}^{2}\right) \mathrm{e}^{-2 \kappa \ell}+\cdots
$$

Minimization of $W_{\pi}(\ell)$ determines the equilibrium MF film thickness $\kappa \ell_{\mathrm{MF}}=$ $\ln \left(-m_{0} / \delta m_{1}\right)$ for $m_{1}<m_{0}$ and shows the standard logarithmic singularity for short-ranged critical wetting as $\delta m_{1} \rightarrow 0^{-}$[2]. Thus, as remarked above, the MF critical wetting phase boundary for the fixed wall magnetization problem is $m_{1}=m_{0}$, corresponding to the vanishing of the first term of the binding potential.

When the interface is no longer planar, FJ determine $m_{\Xi}$ perturbatively by expanding about the planar profile. The original derivation was later simplified by Fisher, Jin and Parry (FJP) [7] who noted that, provided that $\nabla^{2} \ell / \kappa$ and $(\nabla \ell)^{2} \ll 1$, the ansatz

$$
m_{\Xi}(\mathbf{r})=m_{\pi}(z ; \ell(\mathbf{x})) \quad \mathbf{r}=(\mathbf{x}, z)
$$

is an approximate solution to the full Helmholtz equation and exactly satisfies the required boundary conditions. This determines the effective Hamiltonian as

$$
H_{\mathrm{FJ}}[\ell]=\int \mathrm{d} \mathbf{x}\left\{\Sigma_{\alpha \beta}+\frac{\Sigma(\ell)}{2}(\nabla \ell)^{2}+W_{\pi}(\ell)\right\}
$$

up to terms of order $\left(\nabla^{2} \ell\right)$ and $(\nabla \ell)^{4}$. Here $\Sigma(\ell)$ is the position-dependent stiffness coefficient, formally identified as [6]

$$
\Sigma(\ell)=\int_{0}^{\infty} \mathrm{d} z\left(\frac{\partial m_{\pi}(z ; \ell)}{\partial \ell}\right)^{2},
$$

and which is given explicitly in DP approximation by

$$
\Sigma(\ell)=\Sigma_{\alpha \beta}+2 \kappa \delta m_{1} m_{0} \mathrm{e}^{-\kappa \ell}-2 \kappa^{2} \ell m_{0}^{2} \mathrm{e}^{-2 \kappa \ell}+\cdots
$$

where $\Sigma_{\alpha \beta}$ is the stiffness of the free interface (equal to the tension for the present continuum isotropic model).

It is clear that the key ingredient in the derivation of the interfacial Hamiltonian is the identification of the constrained profile $\delta m_{\Xi}=m_{\Xi}-m_{0}$ within the wetting region $(m>0)$. In general, this is a functional of the interfacial configuration (and the wall shape). In anticipation of the non-perturbative derivation presented in the next section, we combine (11) and (15) and note that the FJP ansatz for the magnetization in the wetting layer can be written as two infinite series

$$
\delta m_{\Xi}=\sum_{n=0}^{\infty}\left(\mu_{n} \mathrm{e}^{-\kappa z-n \kappa \ell(\mathbf{x})}-\mu_{n+1} \mathrm{e}^{\kappa z-(n+1) \kappa \ell(\mathbf{x})}\right)
$$

with coefficients

$$
\mu_{n}= \begin{cases}\delta m_{1} & n \text { even }(\text { or } 0) \\ m_{0} & n \text { odd. }\end{cases}
$$

As stated above, this exactly satisfies the boundary conditions and is an approximate solution to the Helmholtz equation, provided that $\nabla^{2} \ell / \kappa$ and $(\nabla \ell)^{2} \ll 1$. Corrections to this expression are of algebraic order in the inverse radii of curvature of the interface.

\section{Non-perturbative derivation}

\subsection{Constrained magnetization with fixed boundary conditions}

To recap, first we require solutions to the Helmholtz equations

$$
\nabla^{2} m_{\Xi}= \begin{cases}\kappa^{2}\left(m_{\Xi}-m_{0}\right), & m_{\Xi}>0 \\ \kappa^{2}\left(m_{\Xi}+m_{0}\right), & m_{\Xi}<0 .\end{cases}
$$


The boundary conditions in the bulk region $\left(m_{\Xi}<0\right)$ are

$$
\left.m_{\Xi}\right|_{\mathbf{r}=\left(\mathbf{x}, \ell(\mathbf{x})^{+}\right)}=0,\left.\quad m_{\Xi}\right|_{z=\infty}=-m_{0}
$$

whilst, within the wetting layer $\left(m_{\Xi}>0\right)$, we must have

$$
\left.m_{\Xi}\right|_{\mathbf{r}=(\mathbf{x}, \psi(\mathbf{x}))}=m_{1},\left.\quad m_{\Xi}\right|_{\mathbf{r}=\left(\mathbf{x}, \ell(\mathbf{x})^{-}\right)}=0 .
$$

We deal with the simpler bulk region $\left(m_{\Xi}<0\right)$ first. Consider the Green's function that solves the Ornstein-Zernike-like equation

$$
\left(-\nabla_{\mathbf{r}_{1}}^{2}+\kappa^{2}\right) K\left(\mathbf{r}_{1}, \mathbf{r}_{2}\right)=2 \kappa \delta\left(\mathbf{r}_{1}-\mathbf{r}_{2}\right)
$$

and which decays to zero for $\left|\mathbf{r}_{1}-\mathbf{r}_{2}\right| \rightarrow \infty$. This has the well-known Yukawa decay

$$
K\left(\mathbf{r}_{1}, \mathbf{r}_{2}\right)=\frac{\kappa}{2 \pi\left|\mathbf{r}_{1}-\mathbf{r}_{2}\right|} \mathrm{e}^{-\kappa\left|\mathbf{r}_{1}-\mathbf{r}_{2}\right|}
$$

and is essentially the Ornstein-Zernike expression for the three-dimensional bulk correlation function. This is not a coincidence and has a direct physical interpretation which we will return to later. Next, in terms of the two-point function, consider the improved ansatz for the constrained profile

$$
m_{\Xi}(\mathbf{r})=-m_{0}+m_{0} \int \mathrm{d} \mathbf{s}_{\ell} K\left(\mathbf{r}_{\ell}, \mathbf{r}\right)
$$

where $\mathbf{r}_{\ell}=(\mathbf{x}, \ell(\mathbf{x}))$ is a point on the interface and $\mathrm{d} \mathbf{s}_{\ell}=\sqrt{1+(\nabla \ell)^{2}} \mathrm{~d} \mathbf{x}$ is the corresponding local area element. At this point we make four remarks:

(A) The ansatz is an exact solution to the Helmholtz partial differential equation (PDE), equation (21), which satisfies the bulk boundary condition, but does not perfectly satisfy the crossing-criterion for all interfacial shapes. However, the error for the latter is exponentially small in the radius of curvature and is therefore irrelevant (see remark C). This contrasts with the FJP ansatz, which exactly satisfies the boundary conditions but is only an approximate solution to the Helmholtz PDE.

(B) For a planar interfacial configuration, the ansatz identically recovers the expression (9) for the planar constrained profile $m_{\pi}(z, \ell)$ in the bulk region $z \geqslant \ell$.

(C) The ansatz satisfies the crossing criterion to exponentially accurate order in the radius of curvature. This is most easily checked for a spherical interfacial configuration of radii $\rho$ which is centred at the origin (say). The details of the wall shape beneath are not a concern, since the constrained profile in the bulk region is independent of this. The exact solution to the Helmholtz problem in this region is the radially symmetric function

$$
m_{\Xi}^{\mathrm{EXACT}}(r)=-m_{0}+m_{0} \frac{\rho}{r} \mathrm{e}^{-\kappa(r-\rho)}
$$

which clearly satisfies the crossing criterion. This compares with the ansatz (26) which, for a spherical configuration, reduces to

$$
m_{\Xi}(r)=-m_{0}+m_{0} \frac{\rho}{r} \mathrm{e}^{-\kappa(r-\rho)}\left(1-\mathrm{e}^{-2 \kappa \rho}\right) .
$$

Whilst not quite exact, the error is completely negligible, since it is exponentially (as opposed to algebraically) small in the radius of the fluid sphere. The same is also true if one considers a constrained cylindrical interfacial shape. Thus, the ansatz will identify correctly any relevant curvature corrections to the effective Hamiltonian.

(D) The integral appearing in the ansatz for the constrained profile has an elegant diagrammatic representation which we have found useful for manipulations and which has an appealing physical interpretation. The two wavy lines represent the wall (lower line) and the $\alpha \beta$ (upper line) interfacial configurations. The thick straight line represents the two-point function 
(Kernel) $K\left(\mathbf{r}_{1}, \mathbf{r}_{2}\right)$, whilst the two circles represent the points $\mathbf{r}_{2}=\mathbf{r}$ (open) and $\mathbf{r}_{1}=\mathbf{r}_{l}$ (black). A black circle (on a surface) means that one must integrate over all positions on that surface with the appropriate infinitesimal area element. Thus, we can rewrite the ansatz (26) via the diagram

$$
m_{\Xi}(r)=-m_{0}+m_{0} \longrightarrow
$$

We now turn attention to the constrained profile within the wetting layer. This will determine the form of the binding potential functional. In light of the above Green's function representation of the profile in the bulk region, consider the infinite series

$$
\delta m_{\Xi}=\sum_{n=0}^{\infty}\left(\mu_{n} I_{w \beta}^{(n)}(\mathbf{r})-\mu_{n+1} I_{\alpha \beta}^{(n)}(\mathbf{r})\right)
$$

where $I_{w \beta}^{(n)}(\mathbf{r})$ and $I_{\alpha \beta}^{(n)}(\mathbf{r})$ represent contributions to the profile arising from the 'tails' of the wall- $\beta$ and $\alpha \beta$ interface, respectively. These are functionals of the interfacial and wall configurations and are more accurate versions of the terms appearing in the FJP expansion (19). They are defined via multi-dimensional integrals over the Green's function $K$ and have the same diagrammatic representation described above. Thus, for the tails of the wall- $\beta$ interface, we have, for the first three terms,

$$
\begin{aligned}
I_{w \beta}^{(1)}(\mathbf{r}) & =\int \mathrm{d} \mathbf{s}_{\psi} K\left(\mathbf{r}_{\psi}, \mathbf{r}\right) \\
I_{w \beta}^{(2)}(\mathbf{r}) & =\iint \mathrm{d} \mathbf{s}_{\ell} \mathrm{d} \mathbf{s}_{\psi} K\left(\mathbf{r}_{\ell}, \mathbf{r}_{\psi}\right) K\left(\mathbf{r}_{\psi}, \mathbf{r}\right) \\
I_{w \beta}^{(3)}(\mathbf{r}) & =\iiint \mathrm{d} \mathbf{s}_{\psi} \mathrm{d} \mathbf{s}_{\ell} \mathrm{d}_{\mathbf{s}^{\prime}}{ }_{\psi} K\left(\mathbf{r}_{\psi}, \mathbf{r}_{\ell}\right) K\left(\mathbf{r}_{\ell}, \mathbf{r}_{\psi}^{\prime}\right) K\left(\mathbf{r}_{\psi}^{\prime}, \mathbf{r}\right)
\end{aligned}
$$

or, equivalently,

$$
I_{w \beta}^{(1)}(\mathbf{r})=\longdiv { \rho }
$$

whilst, for the $\alpha \beta$ interface,

$$
\begin{aligned}
I_{\alpha \beta}^{(1)}(\mathbf{r}) & =\int \mathrm{d} \mathbf{s}_{\ell} K\left(\mathbf{r}_{\ell}, \mathbf{r}\right) \\
I_{\alpha \beta}^{(2)}(\mathbf{r}) & =\iint \mathrm{d} \mathbf{s}_{\psi} \mathrm{d} \mathbf{s}_{\ell} K\left(\mathbf{r}_{\psi}, \mathbf{r}_{\ell}\right) K\left(\mathbf{r}_{\ell}, \mathbf{r}\right) \\
I_{\alpha \beta}^{(3)}(\mathbf{r}) & =\iiint \mathrm{d} \mathbf{s}_{\ell} \mathrm{d} \mathbf{s}_{\psi} \mathrm{d}_{\ell}^{\prime} K\left(\mathbf{r}_{\ell}, \mathbf{r}_{\psi}\right) K\left(\mathbf{r}_{\psi}, \mathbf{r}_{\ell}^{\prime}\right) K\left(\mathbf{r}_{\ell}^{\prime}, \mathbf{r}\right)
\end{aligned}
$$

or,

$$
I_{\alpha \beta}^{(1)}(\mathbf{r})=\longdiv { \gamma }
$$




$$
\begin{aligned}
& I_{\alpha \beta}^{(2)}(\mathbf{r})=20 \\
& I_{\alpha \beta}^{(3)}(\mathbf{r})=20
\end{aligned}
$$

Notice that the value of each corresponding coefficient $\mu_{n}$ in the expansion (30) is simply determined by the location of the extreme black circle (furthest removed from the open circle). The coefficient is $\delta m_{1}$ or $m_{0}$ if it sits on the wall or interface, respectively. Diagrammatically, our ansatz (30) for the constrained profile within the wetting layer is

$$
\begin{aligned}
& \delta m_{\Xi}=\delta m_{1} \widehat{\boldsymbol{Q}}+m_{0} \sqrt{\boldsymbol{C}}+\delta m_{1} \sqrt{\boldsymbol{e}}+\cdots \\
& -\left(m_{0} \widehat{\delta}+\delta m_{1} \widehat{\alpha}+m_{0} \sqrt{\alpha}+\cdots\right) .
\end{aligned}
$$

The expansion is an exact solution to the Helmholtz PDE and identically reduces to the planar constrained profile (10) and (11) if we set $\ell(\mathbf{x})=\ell$ and $\psi=0$. It also satisfies the crossing criterion and wall boundary condition to exponential accuracy in the radii of curvature. Again, this is most easily seen by considering spherical interfacial and wall configurations. We suppose that the wall is a sphere of radius $\mathrm{R}$ and the wetting layer is a sphere of radius $\rho=R+\ell$. Now let the coordinate tend to the wall, $\mathbf{r} \rightarrow \mathbf{r}_{\psi}$, and group the terms in the series according to the same coefficient and the same number of Ks that span the two interfaces:

$\delta m_{\Xi}\left(\mathbf{r}_{\psi}\right)=\delta m_{1} I_{w \beta}^{(1)}\left(\mathbf{r}_{\psi}\right)+m_{0}\left(I_{w \beta}^{(2)}\left(\mathbf{r}_{\psi}\right)-I_{\alpha \beta}^{(1)}\left(\mathbf{r}_{\psi}\right)\right)+\delta m_{1}\left(I_{w \beta}^{(3)}\left(\mathbf{r}_{\psi}\right)-I_{\alpha \beta}^{(2)}\left(\mathbf{r}_{\psi}\right)\right)+\cdots$

Making use of the radial symmetry, the integrals are easily evaluated,

$$
\begin{aligned}
& I_{w \beta}^{(1)}\left(\mathbf{r}_{\psi}\right)=1-\mathrm{e}^{-2 \kappa R} \\
& I_{w \beta}^{(2)}\left(\mathbf{r}_{\psi}\right)-I_{\alpha \beta}^{(1)}\left(\mathbf{r}_{\psi}\right)=1
\end{aligned}
$$

implying that all bar the very first contribution vanishes. In these diagrams, the concentric circles represent the spherical wall and interfacial shapes in an obvious representation, while the open circle represent the fixed point $\mathbf{r}_{\psi}$, which is not integrated over. The first integral is unity (to exponential accuracy in the radius of the wall), giving the correct value of the boundary magnetization. A similar argument holds at the interface. Setting $\mathbf{r}=\mathbf{r}_{\ell}$ and doing the analogous grouping of terms,

$$
\delta m_{\Xi}\left(\mathbf{r}_{\ell}\right)=-m_{0} I_{\alpha \beta}^{(1)}\left(\mathbf{r}_{\ell}\right)-\delta m_{1}\left(I_{\alpha \beta}^{(2)}\left(\mathbf{r}_{\ell}\right)-I_{w \beta}^{(1)}\left(\mathbf{r}_{\ell}\right)\right)-m_{0}\left(I_{\alpha \beta}^{(3)}\left(\mathbf{r}_{\ell}\right)-I_{w \beta}^{(2)}\left(\mathbf{r}_{\ell}\right)\right)+\cdots
$$


with

$$
\begin{aligned}
& I_{\alpha \beta}^{(1)}\left(\mathbf{r}_{\ell}\right)=\varnothing \bigcirc=1-\mathrm{e}^{-2 \kappa(R+\ell)} \\
& I_{\alpha \beta}^{(2)}\left(\mathbf{r}_{\ell}\right)-I_{w \beta}^{(1)}\left(\mathbf{r}_{\ell}\right)=\varnothing 0 \mathrm{e}^{-2 \kappa(R+\ell)} \\
& I_{\alpha \beta}^{(3)}\left(\mathbf{r}_{\ell}\right)-I_{w \beta}^{(2)}\left(\mathbf{r}_{\ell}\right)=\wp\left(0 \mathrm{e}^{-2 \kappa(R+\ell)} .\right.
\end{aligned}
$$

Again, to exponential accuracy, the first integral is equal to unity, while all the other contributions vanish.

\subsection{Non-local binding potential functional with fixed boundary conditions}

Having constructed the constrained profile, the final piece of the derivation is to evaluate $H_{\mathrm{LGW}}\left[m_{\Xi}\right]$. First, we use the divergence theorem to re-express the contribution from the gradient-squared term. Within the present DP approximation, terms arising from integrals over the volume conveniently cancel, so that

$H_{\mathrm{LGW}}\left[m_{\Xi}\right]=-\frac{\delta m_{1}}{2} \int_{\psi} \mathrm{d} \mathbf{s}_{\psi} \nabla m \cdot \mathbf{n}_{\psi}-\frac{m_{0}}{2} \int_{\ell^{-}} \mathrm{d} \mathbf{s}_{\ell} \nabla m \cdot \mathbf{n}_{\ell}-\frac{m_{0}}{2} \int_{\ell^{+}} \mathrm{d} \mathbf{s}_{\ell} \nabla m \cdot \mathbf{n}_{\ell}$

containing only surface terms. Here, $\mathbf{n}_{\psi}$ and $\mathbf{n}_{l}$ are the (local) unit normals at the wall and the interface, respectively, pointing towards the bulk (see figure 1). The utility of this expression does not seem to have been recognised previously. For example, for planar interfacial and wall configurations, this provides a far simpler route to the evaluation of the binding potential. Hence, within DP approximation, we can identify

$W_{\pi}(\ell)=-\frac{\delta m_{1}}{2} \frac{\partial m_{\pi}}{\partial z}(0, \ell)-\frac{m_{0}}{2}\left(\frac{\partial m_{\pi}}{\partial z}\left(\ell^{-}, \ell\right)+\frac{\partial m_{\pi}}{\partial z}\left(\ell^{+}, \ell\right)\right)+\Sigma_{w \beta}+\Sigma_{\alpha \beta}$

which, from (10), recovers the result (13), quoted earlier. For general wall and interfacial configurations, we need to perform three surface integrals. The evaluation of each of these is very similar. Consider the surface integral over the wall

$$
-\frac{\delta m_{1}}{2} \int_{\psi} \mathrm{d} \mathbf{s}_{\psi} \nabla m \cdot \mathbf{n}_{\psi}
$$

Substituting the series expansion for the constrained profile $\delta m_{\Xi}$, equation (30), and grouping the terms together according to coefficients and Kernels, we have

$$
\begin{aligned}
-\frac{\delta m_{1}^{2}}{2} \int_{\psi} \mathrm{d} \mathbf{s}_{\psi} & \nabla I_{w \beta}^{(1)}\left(\mathbf{r}_{\psi}\right) \cdot \mathbf{n}_{\psi}-\frac{m_{0} \delta m_{1}}{2} \int_{\psi} \mathrm{d} \mathbf{s}_{\psi} \nabla\left(I_{w \beta}^{(2)}\left(\mathbf{r}_{\psi}\right)-I_{\alpha \beta}^{(1)}\left(\mathbf{r}_{\psi}\right)\right) \cdot \mathbf{n}_{\psi} \\
& -\frac{\delta m_{1}^{2}}{2} \int_{\psi} \mathrm{d} \mathbf{s}_{\psi} \nabla\left(I_{w \beta}^{(3)}\left(\mathbf{r}_{\psi}\right)-I_{\alpha \beta}^{(2)}\left(\mathbf{r}_{\psi}\right)\right) \cdot \mathbf{n}_{\psi}+\cdots
\end{aligned}
$$

or, diagrammatically,

$$
\begin{aligned}
& -\frac{\delta m_{1}^{2}}{2} \int_{\psi} \mathrm{d} \mathbf{s}_{\psi} \nabla(\widehat{\boldsymbol{\rho}}) \cdot \mathbf{n}_{\psi}-\frac{m_{0} \delta m_{1}}{2} \int_{\psi} \mathrm{d} \mathbf{s}_{\psi} \nabla(\sqrt{\mathbf{0}}) \cdot \mathbf{n}_{\psi} \\
& -\frac{\delta m_{1}^{2}}{2} \int_{\psi} \mathrm{d} \mathbf{s}_{\psi} \nabla\left(\sqrt{\mathbf{c}}-\mathbf{n}_{\psi}+\cdots\right.
\end{aligned}
$$


6442

A O Parry et al

where each gradient is evaluated at the wall, ie. the open circle is placed at the lower wavy line. Ignoring irrelevant terms of order $\exp \left(-\kappa\left(R_{1}^{\psi}(\mathbf{x})+R_{2}^{\psi}(\mathbf{x})\right)\right)$, where $R_{1,2}^{\psi}$ are the principal radii of curvature at the wall, it follows that the scalar field appearing in each gradient term of the expansion is a constant along the surface of the wall, ie.

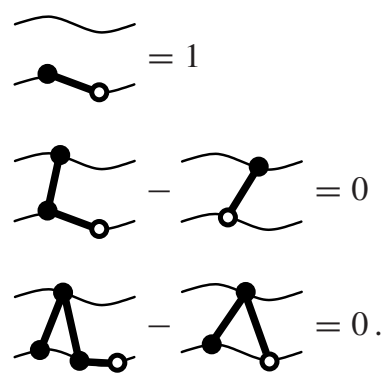

Accordingly, (56) reduces to

$$
\begin{aligned}
& \frac{\delta m_{1}^{2}}{2} \int_{\psi} s_{\psi}|\nabla(\widetilde{z})|+\frac{m_{0} \delta m_{1}}{2} \int_{\psi} d s_{\psi}|\nabla(\mathcal{Z}-J)|
\end{aligned}
$$

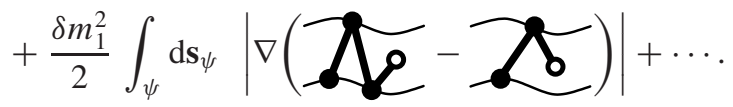

The first term does not describe the wetting behaviour but rather the excess free energy of the wall- $\beta$ interface,

$$
F_{w \beta}[\psi]=\Sigma_{w \beta} \int \mathrm{d} \mathbf{s}_{\psi}+C \int \mathrm{d} \mathbf{s}_{\psi}\left(\frac{1}{R_{1}^{\psi}}+\frac{1}{R_{2}^{\psi}}\right)
$$

where $C=\delta m_{1}^{2}$ is a rigidity modulus. Note that the last term in (61) involves the local mean curvature. No higher-order corrections, containing for example the Gaussian curvature, exist within the present DP approximation. The other terms in the series (60) contribute towards the binding potential functional. For example,

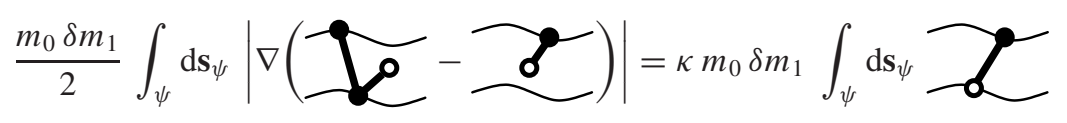

and

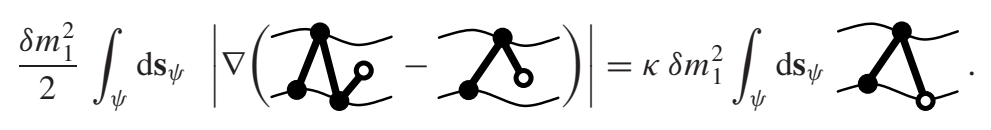

Similar expressions are generated by the surface integrals along the interface. For example, along the bulk side of the interface, where the constrained profile is given by (26) (or equivalently (29)), we find

$$
\begin{array}{r}
-\frac{m_{0}}{2} \int_{\ell^{+}} \mathrm{d} \mathbf{s}_{\ell} \nabla m_{\Xi} \cdot \mathbf{n}_{\ell}=\frac{m_{0}^{2}}{2} \int_{\ell^{+}} \mathrm{d} \mathbf{s}_{\ell} \mid \nabla(\longrightarrow \\
=\frac{\Sigma_{\alpha \beta}}{2} \int_{\ell} \mathrm{d} \mathbf{s}_{\ell}+m_{0}^{2} \int_{\ell} \mathrm{d} \mathbf{s}_{\ell}\left(\frac{1}{R_{1}^{\ell}}+\frac{1}{R_{2}^{\ell}}\right)
\end{array}
$$


where the gradient is evaluated on the upper side of the $\alpha \beta$ interface, i.e. the open circle is placed on the upper wavy line. This expression generates half of the interfacial tension of the $\alpha \beta$ interface and an apparent bending modulus which will later cancel. Here, $R_{1,2}^{\ell}$ are the local radii of curvature of the interface. The final surface integral is along the bottom of the $\alpha \beta$ interface and, using the analogous grouping of terms, we find

$$
\begin{aligned}
&-\frac{m_{0}}{2} \int_{\ell^{-}} \mathrm{d} \mathbf{s}_{\ell} \nabla m_{\Xi} \cdot \mathbf{n}_{\ell}=-\frac{m_{0}^{2}}{2} \int_{\ell^{-}} \mathrm{d} \mathbf{s}_{\ell} \nabla( \\
&-\frac{m_{0} \delta m_{1}}{2} \int_{\ell^{-}} \mathrm{d} \mathbf{s}_{\ell} \nabla( \\
&-\frac{m_{0}^{2}}{2} \int_{\ell^{-}} \mathrm{d} \mathbf{s}_{\ell} \nabla\left(\mathbf{n}_{\ell}\right.
\end{aligned}
$$

The first term is evaluated as

$-\frac{m_{0}^{2}}{2} \int_{\ell^{-}} \mathrm{d} \mathbf{s}_{\ell} \nabla(\widetilde{\boldsymbol{\sigma}}) \cdot \mathbf{n}_{\ell}=\frac{\Sigma_{\alpha \beta}}{2} \int_{\ell} \mathrm{d} \mathbf{s}_{\ell}-m_{0}^{2} \int_{\ell} \mathrm{d} \mathbf{s}_{\ell}\left(\frac{1}{R_{1}^{\ell}}+\frac{1}{R_{2}^{\ell}}\right)$

generating the other half of the interfacial tension and cancelling the bending modulus term in (64). The second term in the expansion simplifies

$$
-\frac{m_{0} \delta m_{1}}{2} \int_{\ell^{-}} \mathrm{d} \mathbf{s}_{\ell} \nabla(\widehat{\mathbf{p}}) \cdot \mathbf{n}_{\ell}=\kappa m_{0} \delta m_{1} \int_{\ell} \mathrm{d} \mathbf{s}_{\ell}
$$

and is the same as (62). Similarly, the third term reduces to

$$
-\frac{m_{0}^{2}}{2} \int_{\ell^{-}} \mathrm{d} \mathbf{s}_{\ell} \nabla(\sqrt{\mathbf{0}}) \cdot \mathbf{n}_{\ell}=\kappa m_{0}^{2} \int_{\ell} \mathrm{d} \mathbf{s}_{\ell}
$$

from which the pattern is apparent. Combining all of the above, the constrained free energy of the wall- $\alpha$ interface can be written as

$$
H_{\mathrm{LGW}}\left[m_{\Xi}\right]=F_{w \beta}[\psi]+H[\ell, \psi]
$$

where our final result for the interfacial Hamiltonian is

$$
H[\ell, \psi]=\Sigma_{\alpha \beta} A_{\alpha \beta}+W[\ell, \psi]
$$

where $A_{\alpha \beta}=\int_{\ell} \mathrm{d} \mathbf{s}_{\ell}$ is the area of the interface. The binding potential functional is exactly given by

$$
W[\ell, \psi]=\sum_{n=1}^{\infty}\left(a_{1} \Omega_{n}^{n}+b_{1} \Omega_{n}^{n+1}+b_{2} \Omega_{n+1}^{n}\right)
$$

with geometry-independent coefficients

$$
\begin{aligned}
& a_{1} \equiv 2 \kappa m_{0} \delta m_{1}=\sqrt{8 \Sigma_{w \beta} \Sigma_{\alpha \beta}} \\
& b_{1} \equiv \kappa m_{0}^{2}=\Sigma_{\alpha \beta} \\
& b_{2} \equiv \kappa \delta m_{1}^{2}=2 \Sigma_{w \beta} .
\end{aligned}
$$

Equation (71) is the main result of our paper, and expresses the binding potential functional as a sum of terms $\Omega_{\mu}^{v}[\ell, \psi]$, which are each multiple integrals over the Green's function $K$ connecting $\mu$ points on the wall with $v$ points on the interface. They have the same 
diagrammatic representation described earlier but contain no open circles. Thus the three leading-order terms, corresponding to $n=1$ in the series, are

$$
\begin{aligned}
\Omega_{1}^{1}[\ell, \psi] & =\iint \mathrm{d} \mathbf{s}_{\psi} \mathrm{d} \mathbf{s}_{\ell} K\left(\mathbf{r}_{\psi}, \mathbf{r}_{\ell}\right)= \\
\Omega_{1}^{2}[\ell, \psi]= & \iiint \mathrm{d} \mathbf{s}_{\ell} \mathrm{d} \mathbf{s}_{\psi} \mathrm{d} \mathbf{s}_{\ell}^{\prime} K\left(\mathbf{r}_{\ell}, \mathbf{r}_{\psi}\right) K\left(\mathbf{r}_{\psi}, \mathbf{r}_{\ell}^{\prime}\right) \\
= & \int \mathrm{d} \mathbf{s}_{\psi}\left[\int \mathrm{d} \mathbf{s}_{\ell} K\left(\mathbf{r}_{\psi}, \mathbf{r}_{\ell}\right)\right]^{2} \\
\Omega_{2}^{1}[\ell, \psi]= & \iiint \mathrm{d} \mathbf{s}_{\psi} \mathrm{d} \mathbf{s}_{\ell} \mathrm{d} \mathbf{s}_{\psi}^{\prime} K\left(\mathbf{r}_{\psi}, \mathbf{r}_{\ell}\right) K\left(\mathbf{r}_{\ell}, \mathbf{r}_{\psi}^{\prime}\right) \\
& =\int \mathrm{d} \mathbf{s}_{\ell}\left[\int \mathrm{d} \mathbf{s}_{\psi} K\left(\mathbf{r}_{\ell}, \mathbf{r}_{\psi}\right)\right]^{2}=
\end{aligned}
$$

These diagrams were identified and discussed in [1], and are responsible for the leading-order terms in the binding potential (14) (see below). The present derivation also identifies higherorder terms in the expansion of the binding potential functional. For example, the three $n=2$ terms are represented diagrammatically by

$$
\Omega_{2}^{2}[\ell, \psi]=
$$

and similarly for larger values of $n$. Note that all the diagrams correspond to planar graphs and have a simple 'lace' or 'zig-zag' form in which the black circles are sequentially connected by a single thick line, each representing a Green's function $K$. As we shall see, these higher-order diagrams $(n>1)$ are responsible for the hard-wall repulsion in the binding potential functional (and function).

\section{Binding potential functions and position-dependent stiffnesses}

Having derived the explicit form of the non-local model, we show that it reproduces the known form of the binding potential for planar and spherical interfacial configurations and, in addition, recovers the FJ Hamiltonian with its position-dependent stiffness coefficient in the small-gradient limit.

\subsection{Planar interfaces}

Consider the simplest case of a planar wall and a planar interface of constant thickness: $\psi=0$, $\ell(\mathbf{x})=\ell$. Let $A_{w}$ denote the area of the wall (and interface). When evaluated at $\ell(\mathbf{x})=\ell$, the binding potential functional per unit area necessarily reduces to the planar binding potential

$$
W_{\pi}=\left.\frac{W[\ell, 0]}{A_{w}}\right|_{\ell(\mathbf{x})=\ell} .
$$


The contributions to the functional are easily evaluated. At leading order $n=1$,

$$
\begin{aligned}
& \Omega_{1}^{1}=\longrightarrow=A_{w} \mathrm{e}^{-\kappa \ell} \\
& \Omega_{1}^{2}=A_{w} \mathrm{e}^{-2 \kappa \ell} \\
& \Omega_{2}^{1}=\longrightarrow=A_{w} \mathrm{e}^{-2 \kappa \ell}
\end{aligned}
$$

implying

$$
W_{\pi}(\ell)=a_{1} \mathrm{e}^{-\kappa \ell}+\left(b_{1}+b_{2}\right) \mathrm{e}^{-2 \kappa \ell}+\cdots
$$

which recovers the traditional form of the binding potential function in theories of critical wetting, equation (14).

The higher-order terms in the functional can also be easily evaluated:

$$
\begin{aligned}
& \Omega_{n}^{n}=A_{w} \mathrm{e}^{-(2 n-1) \kappa \ell} \\
& \Omega_{n}^{n+1}=A_{w} \mathrm{e}^{-2 n \kappa \ell} \\
& \Omega_{n+1}^{n}=A_{w} \mathrm{e}^{-2 n \kappa \ell} .
\end{aligned}
$$

Note that, within the binding potential functional (71), each of the $\Omega_{n}^{n}$ enters with the same coefficient, which is also the case for $\Omega_{n}^{n+1}$ and $\Omega_{n+1}^{n}$. A trivial resummation then leads to

$$
W_{\pi}=a_{1} \frac{\mathrm{e}^{-\kappa \ell}}{1-\mathrm{e}^{-2 \kappa \ell}}+\left(b_{1}+b_{2}\right) \frac{\mathrm{e}^{-2 \kappa \ell}}{1-\mathrm{e}^{-2 \kappa \ell}}
$$

which is identical to the known result quoted earlier, equation (13).

The hard-wall divergence of this function as $\ell \rightarrow 0$ is, therefore, directly related to the higher-order diagrams in the binding potential functional.

\subsection{Spherical interfaces}

A similar calculation can be performed for the problem of wetting around a sphere (and cylinder) with interesting consequences. We suppose that the sphere is of radius $R$ and consider an interfacial configuration corresponding to a sphere of radius $R+\ell$. This class of configurations is sufficient to recover the MF result for the interfacial thickness - a problem which continues to attract attention $[15,16]$. by

The three leading-order terms appearing in the binding potential functional are now given

$$
\begin{aligned}
& \Omega_{1}^{1}=\left(3=\sqrt{A_{w} A_{\alpha \beta}} \mathrm{e}^{-\kappa \ell}\right. \\
& \Omega_{2}^{1}=0 A_{w} \mathrm{e}^{-2 \kappa \ell} \\
& \Omega_{1}^{2}=0=A_{\alpha \beta} \mathrm{e}^{-2 \kappa \ell}
\end{aligned}
$$


where $A_{\alpha \beta}=4 \pi(\ell+R)^{2}$ is the surface area of the interface. More generally, the higher-order diagrams are given by

$$
\begin{aligned}
& \Omega_{n}^{n}=\sqrt{A_{w} A_{\alpha \beta}} \mathrm{e}^{-(2 n-1) \kappa \ell} \\
& \Omega_{n}^{n+1}=A_{\alpha \beta} \mathrm{e}^{-2 n \kappa \ell} \\
& \Omega_{n+1}^{n}=A_{w} \mathrm{e}^{-2 n \kappa \ell} .
\end{aligned}
$$

In writing these, we have ignored terms of order $\mathrm{e}^{-2 \kappa R}$, which are certainly negligible for spheres of mesoscopic size, pertinent to studies of wetting. This point is perhaps worth emphasizing. The equilibrium thickness of a wetting layer around a sphere at and above the wetting temperature is of order $(1 / \kappa) \ln R$, which is a result valid both at MF level and beyond. Ignoring terms or order $\mathrm{e}^{-2 \kappa R}$ is therefore equivalent to neglecting terms of order $\exp \left(-\mathrm{e}^{\kappa \ell}\right)$ in the free energy. Returning to our proof, the different terms in the functional can be resummed and, combining (61), (69) and (71), the constrained excess free energy of this wall- $\beta$ interface reduces to

$$
F_{w \alpha}[\ell]=F_{w \beta}+\Sigma_{\alpha \beta} A_{\alpha \beta}+A_{w} W_{s}(\ell)
$$

where

$$
\frac{F_{w \beta}}{A_{w}}=\frac{\kappa \delta m_{1}^{2}}{2}\left(1+\frac{1}{\kappa R}\right)
$$

and the spherical binding potential

$$
A_{w} W_{s}(\ell)=a_{1} \sqrt{A_{\alpha \beta} A_{w}} \frac{\mathrm{e}^{-\kappa \ell}}{1-\mathrm{e}^{-2 \kappa \ell}}+\left(b_{1} A_{\alpha \beta}+b_{2} A_{w}\right) \frac{\mathrm{e}^{-2 \kappa \ell}}{1-\mathrm{e}^{-2 \kappa \ell}} .
$$

The last two expressions are the known results as calculated directly from the Landau theory within the DP approximation (for fixed boundary magnetization) [16]. Note that each exponential contribution contains polynomial corrections due to the thickness dependence of the interfacial area $A_{\alpha \beta}$. The binding potential function for spheres is therefore different to the planar interfacial binding potential — a result that has not always been recognized. Indeed, the manner in which the area of the unbinding interface enters the form of the binding potential may be viewed as a subtle signature of non-local effects at short-ranged wetting. The influence of non-locality on the equilibrium film thickness and the total mass adsorption may well be observable in density functional studies.

\subsection{Origin of the position-dependent stiffness coefficient}

Within the non-local model there is no explicit position dependence to the surface tension. Despite this, the non-local model identically recovers the FJ Hamiltonian in the small gradient limit and generates an effective position-dependent stiffness from the non-local nature of the binding potential functional. This naturally explains why the coefficients appearing in the FJ stiffness are the same as those appearing in the binding potential function. To this end, we focus on a planar wall $(\psi=0)$ and analyse the structure of the dominant $(n=1)$ terms in the binding potential functional when $\nabla \ell$ is small. Thus, we write

$$
W[\ell, 0]=a_{1} \Omega_{1}^{1}+b_{1} \Omega_{1}^{2}+b_{2} \Omega_{2}^{1}+\cdots
$$

where $a_{1}, b_{1}$ and $b_{2}$ are given by (72). Diagrammatically, this is

$$
W[\ell, 0]=a_{1} \longrightarrow+b_{1} \longrightarrow+b_{2} \precsim+
$$


The results are best expressed in terms of the transverse Fourier transform of the Green's function $K$ which, recall, is simply proportional to the bulk correlation function. We define

$$
K(\ell ; Q)=\int \mathrm{d} \mathbf{x} \frac{\kappa \mathrm{e}^{-\kappa \sqrt{x^{2}+\ell^{2}}}}{2 \pi \sqrt{x^{2}+\ell^{2}}} \mathrm{e}^{\mathrm{ix} \cdot \mathbf{Q}}
$$

which can be evaluated explicitly:

$$
K(\ell ; Q)=\frac{\kappa}{\sqrt{\kappa^{2}+Q^{2}}} \mathrm{e}^{-\sqrt{\kappa^{2}+Q^{2}} \ell} .
$$

It is also convenient to introduce the moment expansion

$$
K(\ell ; Q)=K_{0}(\ell)+Q^{2} K_{2}(\ell)+\cdots
$$

so that we can identify the zeroth and second moments

$$
K_{0}(\ell)=\mathrm{e}^{-\kappa \ell}
$$

and

$$
K_{2}(\ell)=-(1+\kappa \ell) \frac{\mathrm{e}^{-\kappa \ell}}{2 \kappa^{2}}
$$

respectively. These moments of the bulk correlation function will determine the position dependence of the planar binding potential and stiffness.

As discussed above, when the interface is perfectly flat, the integrals determining $\Omega_{1}^{1}, \Omega_{1}^{2}$ and $\Omega_{2}^{1}$ are particularly simple, and we may write

$$
W_{\pi}(\ell)=a_{1} K_{0}(\ell)+\left(b_{1}+b_{2}\right) K_{0}(\ell)^{2}+\cdots
$$

which is the same as (83), quoted above.

Next, suppose that the interface is non-planar. Holding the point on the interface fixed, and doing the integral over the wall first, both $\Omega_{1}^{1}$ and $\Omega_{2}^{1}$ generate effectively local interactions

$$
\begin{aligned}
& \longrightarrow \mathrm{d} \mathbf{x} \sqrt{1+\nabla \ell(\mathbf{x})^{2}} \mathrm{e}^{-\kappa \ell(\mathbf{x})} \\
& \boldsymbol{Z}=\int \mathrm{d} \mathbf{x} \sqrt{1+\nabla \ell(\mathbf{x})^{2}} \mathrm{e}^{-2 \kappa \ell(\mathbf{x})} .
\end{aligned}
$$

These clearly generate an effective position dependence to the stiffness coefficient. Indeed, equation (99) is responsible for the leading-order exponential term in the FJ expression (18). The contribution to the effective position-dependent stiffness from $\Omega_{2}^{1}$ is not important, since it is only $\mathcal{O}\left(\mathrm{e}^{-2 \kappa \ell}\right)$.

The negative decaying term $\propto \ell \mathrm{e}^{-2 \kappa \ell}$ in the FJ result (18) arises from the $\Omega_{1}^{2}$ contribution

$$
\mathcal{Y}=\int \mathrm{d} \mathbf{x}_{1}\left\{\int \mathrm{d} \mathbf{x}_{2} \sqrt{1+\nabla \ell\left(\mathbf{x}_{2}\right)^{2}} K\left(r_{12}\right)\right\}^{2}
$$

which remains non-local. Formally, however, if we assume that the gradient $\nabla \ell$ is small, one may Taylor expand the value of $\ell\left(\mathbf{x}_{2}\right)$ about each point $\mathbf{x}_{1}$ and obtain

$$
\widetilde{\gamma} \approx \int \mathrm{d} \mathbf{x} \mathrm{e}^{-2 \kappa \ell(\mathbf{x})}+\int \mathrm{d} \mathbf{x} S(\ell(\mathbf{x}))(\nabla \ell)^{2}+\cdots
$$

where

$$
S(\ell)=K_{0}(\ell)^{2}+2 K_{0}^{\prime}(\ell) K_{2}^{\prime}(\ell)
$$


yielding

$$
S(\ell)=-\kappa \ell \mathrm{e}^{-2 \kappa \ell}+\cdots
$$

The first term in the expansion (102) generates the second-order contribution $b_{1} \mathrm{e}^{-2 \kappa \ell}$ in the planar binding potential. The second integral generates another contribution to the effective position-dependent stiffness. The presence of the second moment of the bulk correlation function is significant, since it is not a pure exponential and is negative. Gathering together the above, we find, to square-gradient order,

$$
H[\ell, 0] \approx \int \mathrm{d} \mathbf{x}\left\{\Sigma_{\alpha \beta}+\frac{\Sigma(\ell)}{2}(\nabla \ell)^{2}+W_{\pi}(\ell)\right\}
$$

with binding potential $W_{\pi}(\ell)$ given by (83), and the effective position-dependent stiffness coefficient

$$
\Sigma(\ell)=\Sigma_{\alpha \beta}+a_{1} \mathrm{e}^{-\kappa \ell}-2 b_{1} \kappa \ell \mathrm{e}^{-2 \kappa \ell}+\cdots
$$

where the dots represent sub-dominant terms of order $\mathrm{e}^{-2 \kappa \ell}$, which can be ignored. This is the same as the FJ Hamiltonian, and identifies a hitherto unseen connection between the coefficients appearing in the binding potential and position-dependent stiffness.

Finally, we note that, when both the interface and wall are non-planar, all the contributions $\Omega_{\mu}^{v}[\ell, \psi]$ to the binding potential functional are non-local. In the small-gradient limit, $|\nabla \ell|$, $|\nabla \psi| \ll 1$, one may expand $\Omega_{1}^{1}$ and $\Omega_{2}^{1}$, and derive the approximate local limit

$$
H[\ell, \psi]=\left(\Sigma_{\alpha \beta}+\Sigma_{w \beta}\right) \int \mathrm{d} \mathbf{x}+\Delta H[\ell, \psi]
$$

where the interaction part of the Hamiltonian is

$$
\begin{aligned}
\Delta H[\ell, \psi]= & \int \mathrm{d} \mathbf{x}\left\{\frac{\Sigma(\ell-\psi)}{2}(\nabla \ell)^{2}+\Sigma_{12}(\ell-\psi)(\nabla \ell \cdot \nabla \psi)\right. \\
& \left.+\frac{\Sigma_{w \beta}(\ell-\psi)}{2}(\nabla \psi)^{2}+W_{\pi}(\ell-\psi)\right\} .
\end{aligned}
$$

This introduces two more effective position-dependent stiffness coefficients, which depend on the local relative displacement of the interface and wall. The coefficient of $(\nabla \psi)^{2} / 2$ is the position-dependent stiffness of the wall- $\beta$ interface and is given by

$$
\Sigma_{w \beta}(\ell)=\Sigma_{w \beta}+a_{1} \mathrm{e}^{-\kappa \ell}-2 b_{2} \kappa \ell \mathrm{e}^{-2 \kappa \ell}+\cdots
$$

The origin of this term is exactly analogous to the FJ stiffness $\Sigma(\ell)$, discussed above, except that it is now the $\Omega_{2}^{1}$ term that determines the coefficient of $\mathrm{e}^{-2 \kappa \ell}$.

The effective stiffness associated with the off-diagonal term $\nabla \ell \cdot \nabla \psi$ is more intriguing, and is dominated by the non-locality of $\Omega_{1}^{1}$. Again, this is related to the second moment of the Green's function:

$$
\Sigma_{12}(\ell)=-a_{1} K_{2}^{\prime \prime}(\ell)=\frac{a_{1}}{2} \kappa \ell \mathrm{e}^{-\kappa \ell}+\cdots
$$

The Hamiltonian (108) and the stiffness coefficients are precisely the same as those derived by Boulter and Parry [17] and Rejmer and Napiorkowski [18]. The effective position dependence of $\Sigma_{12}$ plays an important role in the theory of surface correlation functions and their sum-rules at complete wetting [19], and owes its origin to the non-locality of $\Omega_{1}^{1}$. 


\section{Discussion}

In this paper, we have shown how a non-local interfacial model for short-ranged wetting phenomena in three dimensions can be derived from an underlying LGW Hamiltonian. Our main result is an expression for the non-local binding potential functional, which is accurate to exponential good order in the radii of curvature of the interface and wall. The binding potential functional, derived for boundary conditions corresponding to fixed surface magnetization, and within the DP approximation, has an elegant diagrammatic expansion

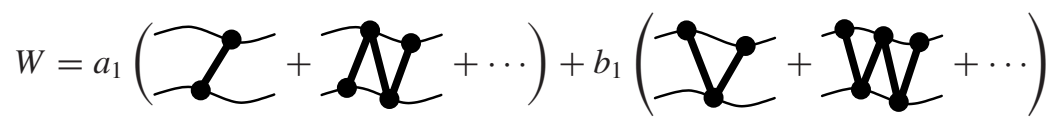

$$
\begin{aligned}
& +b_{2}(20)
\end{aligned}
$$

where each planar graph corresponds to a functional $\Omega_{v}^{\mu}[\ell, \psi]$ connecting $v$ points at the wall with $\mu$ points at the interface via a simple 'zig-zag'. The coefficients $a_{1}, b_{1}$ and $b_{2}$ are determined explicitly and are geometry independent. This expression exactly recovers the full form of the simpler binding potential function for planar and spherical interfacial configurations and explains the origin of the interfacial area dependence in these potentials. This has not been recognized previously.

The non-local Hamiltonian describes a host of different interfacial phase transitions occurring in different geometries. The most well-studied example is the critical wetting transition occurring at a planar wall. The critical singularities of this transition are controlled by the two leading-order terms in the functional, so that one may approximate

$$
W[\ell, \psi] \approx a_{1} \longrightarrow+b_{1}
$$

together with a hard-wall repulsion, which mimics the effect of the higher-order diagrams. This is the same as the model studied in [1]. The non-locality of the $\Omega_{1}^{2}$ interaction appears to be crucial for a description of critical wetting. Whilst the model (and the RG flow equations) recover the model of FJ in the small-gradient limit, non-perturbative effects arise in the RG flow. In particular, there is no stiffness instability mechanism [8] and the transition remains continuous in the presence of large-scale interfacial fluctuation effects. However, whilst the asymptotic criticality has the same non-universal character as in the very simplest capillarywave model [3], there are no significant deviations from mean-field-like criticality for certain observables (surface susceptibility, etc) until the wetting film is rather thick and the area of the wall is very large. This appears to comply with the findings of Ising model Monte Carlo simulation studies [4].

Another geometry in which non-locality manifests itself directly is the linear wedge, where numerical studies based on the LGW model reveal hidden relations (wedge covariance) between wetting and filling which are naturally explained by the non-local theory [9].

To finish our article, we make a number of critical remarks. Firstly, the present derivation is due for some criticism, since it is based on a model with fixed surface magnetization, and it is limited to a DP bulk interaction potential $\Delta \phi$. Whilst previous experience with wetting transitions is strongly suggestive that these features do not influence the underlying physics, it is important to check this quantitatively. This is the topic of our second paper, where we extend the Green's function method to consider (a) perturbations about the DP approximation, (b) the inclusion of squared Laplacian terms in the LGW model, and (c) coupling to a surface field and enhancement. 
One may also remark that alternative choices for the definition of the location of the $\alpha \beta$ interface are possible. FJ, for example, introduce a number of integral criterion definitions which were later refined by Boulter and Indekeu [20]. These alternative definitions do not alter significantly the form of the binding potential function. It is likely that this is also the case for the non-local functional.

Following on from the above two comments, the structure of the non-local functional is in fact largely necessitated by exact statistical mechanic sum-rules. The binding potential functional is essentially the same as the equilibrium free energy $F[\ell, \psi]$ of a thin (magnetic) film bounded by walls fixed at locations described by the fields $\ell(\mathbf{x})$ and $\psi(\mathbf{x})$, at which surface fields $h_{2}$ and $h_{1}$ act, respectively. Taking derivatives of the free energy with respect to the surface fields, generate sum-rules which relate response functions to integrals over the connected spin-spin correlation function $G\left(\mathbf{r}_{1}, \mathbf{r}_{2}\right)$. Thus, for example,

$$
\frac{\partial^{2} F[\ell, \psi]}{\partial h_{1} \partial h_{2}}=k_{\mathrm{B}} T \iint \mathrm{d} \mathbf{s}_{\psi} \mathrm{d} \mathbf{s}_{\ell} G\left(\mathbf{r}_{\ell}, \mathbf{r}_{\psi}\right)
$$

where, as earlier, $\mathbf{r}_{\ell}$ and $\mathbf{r}_{\psi}$ denote points at the interface and wall, respectively. If the thickness of the thin film is much greater than $1 / \kappa$, the function $G$ appearing in the above formula will be essentially the bulk Ornstein-Zernike correlation function, in which case the right-hand side reduces immediately to the functional $\Omega_{1}^{1}$. Similarly, sum-rules for $\partial^{2} F / \partial h_{1}^{2}$ and $\partial^{2} F / \partial h_{2}^{2}$ give rise to $\Omega_{1}^{2}$ and $\Omega_{2}^{1}$-like contributions. These sum-rules considerations are strongly suggestive that the structure of the non-local functional is robust.

The non-local Hamiltonian can also be used to study adsorption phenomena in geometries for which local Hamiltonians cannot be applied due to ambiguities concerning the way in which the local interaction with the wall is measured. An example of this is the case of complete wetting in a parabola or paraboloid. Macroscopic arguments show that the adsorption isotherms must show some non-trivial cross-over behaviour when the radii of curvature of the meniscus and the wall are similar [21]. This phenomenon is certainly experimentally observable [22] and one would like to be able to understand it at a more microscopic level. The present non-local model provides a far simpler method of studying this than density functional theory.

Finally, we point out that the diagrammatic expansion (111) lends itself towards a rather physical interpretation of the origin of the binding potential functional. The essential ingredient in the construction of the functional is the Green's function $K$, which is simply proportional to the bulk three-dimensional correlation function. Consequently, each time we encounter a thick line connecting two points in a planar graph, one should think of this representing a bulk-like correlation between the magnetization at both points. It is natural at this point to invoke the bubble or wandering solid-on-solid tube model of low-temperature correlations, which has been applied very successfully by Abraham, Fisher and co-workers [23, 24]. According to this picture, the correlation function between two points in a phase of net positive magnetization below the critical point arises due to a bubble (in two dimensions) or tube (in three dimensions) of net negative magnetization which connects the two points and is subject to thermal fluctuations. If we adopt this picture, each thick line in a planar graph appears to represent a contribution to the free energy arising from the wandering of a pseudo-onedimensional tube of the bulk ( $\alpha$ phase) which tunnels from the interface to the wall. Higherorder diagrams account for reflections of the wandering tube from either of the surfaces.

\section{Acknowledgments}

CR acknowledges support from the Dirección General de Investigación-MCyT (BFM200300180) and the Comunidad de Madrid (S-0505/ESP/0299). NRB acknowledges support from the Portuguese Foundation for Science and Technology (SFRH/BD/16424/2004). JMR-E acknowledges support from the European Commission (MEIF-CT-2003-501042). 


\section{References}

[1] Parry A O, Romero-Enrique J M and Lazarides A 2004 Phys. Rev. Lett. 93086104

[2] Dietrich S 1988 Phase Transitions and Critical Phenomena vol 12, ed C Domb and J L Lebowitz (New York: Academic)

See, also Schick M 1990 Liquids at Interfaces ed J Charvolin, J F Joanny and J Zinn-Justin (Amsterdam: Elsevier) p 3364

[3] Brézin E, Halperin B I and Leibler S 1983 Phys. Rev. Lett. 501387

Fisher D S and Huse D A 1985 Phys. Rev. B 32247

[4] Binder K, Landau D P and Kroll D M 1986 Phys. Rev. Lett. 562272

Binder K, Landau D P and Wansleben S 1989 Phys. Rev. B 406979

[5] Gompper G and Kroll D M 1988 Phys. Rev. B 373821

[6] Fisher M E and Jin A J 1991 Phys. Rev. B 441430

Jin A J and Fisher M E 1993 Phys. Rev. B 477365

[7] Fisher M E, Jin A J and Parry A O 1994 Bunsenges. Phys. Chem. 98357

[8] Fisher M E and Jin A J 1992 Phys. Rev. Lett. 69792

Jin A J and Fisher M E 1993 Phys. Rev. B 482642

Boulter C J 1997 Phys. Rev. Lett. 791897

[9] Greenall M G, Parry A O and Romero-Enrique J M 2004 J. Phys.: Condens. Matter 162515

[10] Rejmer K, Dietrich S and Napiorkowski M 1999 Phys. Rev. E 604027

[11] Balian R and Bloch C 1970 Ann. Phys. 60401

[12] Adagideli I, Sheehy D E and Goldbart P M 2002 Int. J. Mod. Phys. 161381

[13] Kac M 1966 Am. Math. Monthly 731

[14] Holyst R and Poniewierski A 1987 Phys. Rev. B 365628

Gelfand M P and Lipowsky R 1987 Phys. Rev. B 368725

[15] Upton P J, Indekeu J O and Yeomans J 1989 Phys. Rev. B 40666

Gil T and Mikheev L B 1995 Phys. Rev. E 52772

Hadjiagapiou I A 1996 J. Chem. Phys. 1052927

Bieker T and Dietrich S 1998 Physica A 25285

Evans R, Roth R and Bryk P 2003 Europhys. Lett. 62815

Evans R, Henderson J R and Roth R 2004 J. Chem. Phys. 12112074

Steward M C and Evans R 2005 Phys. Rev. E 71011602

[16] Parry A O, Rascón C and Morgan L 2006 J. Chem. Phys. 124151101

[17] Boulter C and Parry A O 1995 Phys. Rev. Lett. 742403

[18] Rejmer K and Napiorkowski M 1995 Z. Phys. B 97293

[19] Parry A O and Boulter C J 1995 Physica A 21877

[20] Boulter C J and Indekeu J O 1997 Phys. Rev. E 565734

[21] Rascón C and Parry A O 2000 Nature 407986

[22] Bruschi L et al 2006 at press

[23] Abraham D B 1983 Phys. Rev. Lett. 50291

Fisher M E 1984 J. Stat. Phys. 34667

Abraham D B et al 1992 Phys. Rev. Lett. 68423

[24] Abraham D B, Chayes J T and Chayes L 1984 Commun. Math. Phys. 96439 